\title{
Desenvolvimento Tecnológico e a Maturidade das Pesquisas no Âmbito das Instituições de Pesquisa Científica e Tecnológica (ICT) no Brasil
}

\author{
Technological Development and Maturity of Research in the Field of \\ Scientific and Technological Research Institutions (TRI) in Brazil
}

\author{
Grace Ferreira Ghesti ${ }^{1}$ \\ Larisse Araújo Lima ${ }^{1}$ \\ Lincoln Pinheiro de Oliveira ${ }^{1}$ \\ Luiza Xavier da Silva Tenório ${ }^{1}$ \\ Marcio Lima da Silva ${ }^{1}$ \\ Sarah Sampaio Py-Daniel ${ }^{1}$ \\ Thiago Lara Fernandes ${ }^{1}$ \\ ${ }^{1}$ Universidade de Brasília (UnB) CDT, Brasilia, DF, Brasil
}

\begin{abstract}
Resumo
O desenvolvimento tecnológico oriundo de Instituições de Pesquisa Científica e Tecnológica (ICT) tem se caracterizado como um desafio junto ao processo de inovação. Se por um lado o país conta com o apoio das ICTs para promover o contínuo crescimento tecnológico e o desenvolvimento de novas tecnologias que preencham o gap mercadológico, por outro lado, o país experimenta as dificuldades dos entraves no desenvolvimento das pesquisas. Percebe-se que a escassez de recursos e a carência de apoio do setor industrial em tecnologias em estágios iniciais são algumas das barreiras encontradas por pesquisadores. O presente estudo analisou que aproximadamente $75 \%$ das tecnologias se estabelecem nas pesquisas básicas. Como fruto desse estudo observou-se ainda que os investimentos governamentais $e$ as parcerias com a indústria para o desenvolvimento de novas tecnologias são escassos. Contudo, esforços direcionados a junção entre ICTs, setor empresarial e governo podem contribuir para um futuro promissor e um contínuo crescimento do país.
\end{abstract}

Palavras-chave: Maturidade Tecnológica. Pesquisa. Universidade.

\begin{abstract}
The technological development coming from the Institutions of Scientific and Technological Research (TRI) has been characterized as a challenge with the innovation process. While on the one hand the country counts on the support of the ICTs to promote the continuous technological growth and the development of new technologies that suppress the market gap, on the other hand these experience the difficulties of the obstacles in the development of the researches. Scarcity of resources and lack of support from the industrial sector in technologies in the early stages are some of the barriers encountered by researchers. The present study analyzed that about $75 \%$ are established in basic research. As a result of this study it was observed that government investments and partnerships with industry for the development of new technologies are scarce. However, efforts aimed at bringing together ICTs, the business sector and government can contribute to the country's promising future and continued growth.
\end{abstract}

Keywords: Technological Maturity. Search. University.

Área Tecnológica: Desenvolvimento Científico e Tecnológico. Prospecção Tecnológica. 


\section{Introdução}

O atual cenário de inovação no Brasil surge como um campo com relevantes possibilidades de exploração. Grande parte do desenvolvimento tecnológico gerado no país é proveniente das Instituições de Pesquisa Científica e Tecnológica (ICT); entretanto, as pesquisas desenvolvidas no âmbito das ICTs, em especial nas universidades, se caracterizam como deficitárias em relação à maturidade tecnológica e, consequentemente, em relação à disponibilização de seu produto final para o mercado consumidor (TURCHI; MORAIS, 2017; GESTIC, 2017). Dentro desse contexto, é possível observar que as tecnologias são idealizadas, iniciam seu processo de desenvolvimento e estagnam nos momentos iniciais de prontidão tecnológica (GESTIC, 2017).

De acordo com Massambani (2017), as tecnologias desenvolvidas no âmbito das ICTs em sua maioria, em torno de $75 \%$, se estabelecem nas pesquisas básicas, ou seja, se encontram em uma pesquisa de baixo a médio impacto e que repercute apenas em difusão tecnológica para inovação. Aproximadamente $23 \%$ encontram-se nas pesquisas aplicadas, compreendendo as pesquisas de médio impacto e repercutindo em tecnologias com pré-investimentos e inovação para desempenho. E apenas 3\% se destacam em pesquisas de alto impacto, correspondendo a pesquisas aplicadas, demonstrações e pilotos; com repercussão em inovação para competitividade, inovação pioneira ou inovação crítica.

O motivo para esse fenômeno de paralisação do desenvolvimento pode ser atribuído a diversos fatores: burocracia nos trâmites administrativos para realização de pesquisas avançadas; falta de recursos para custeio de novas pesquisas ou mesmo a falta de interesse do parque industrial em investir em tecnologias em estágios iniciais são algumas das barreiras encontradas por pesquisadores dentro das ICTs (SENADO FEDERAL, 2016).

Segundo Lozardo (2017 apud TURCHI; MORAIS, 2017), presidente do Instituto de Pesquisa Econômica Aplicada (IPEA), os investimentos e as iniciativas em apoio à educação, à ciência, à tecnologia e à inovação são fundamentais para subsidiar políticas de superação da estagnação da produtividade dos fatores de produção nacionais e, ademais, para promover o desenvolvimento econômico e social de longo prazo. Tais iniciativas podem compreender ações que permitam proporcionar condições de subsistência ao desenvolvimento das pesquisas, por exemplo, o apoio das agências de fomento no tocante às ações de promoção ao desenvolvimento científico, tecnológico e de inovação; o apoio aos processos de transferência de tecnologia e as parcerias entre empresas e ICTs (FINEP, 2018b; GESTIC, 2017). Além disso, a participação de parques tecnológicos e de incubadoras nos processos de desenvolvimento vem despontado como estratégia eficaz e promissora no meio acadêmico (GESTIC, 2017).

A busca por parcerias que possibilitem superar esse processo de estagnação sobrevém justamente por meio de investimentos que proporcionem elevar a maturidade tecnológica das pesquisas de base. A maturidade tecnológica, por sua vez, segue padrões ou níveis que permitem analisar o quão pronto está o produto para o consumidor final, deixando assim de ser meramente uma ideia concebida a respeito de um gap do mercado para uma tecnologia completa e estruturada (GESTIC, 2017).

De acordo com Turchi e Morais (2017), nas duas últimas décadas, o Estado brasileiro intensificou esforços na consolidação do Sistema Nacional de Inovação (SNI), com o objetivo de ampliar o apoio e a promoção das atividades de ciência, tecnologia e inovação (CT\&I) no país. Para isso, o processo de fortalecimento do SNI se baseou na formação de pesquisadores, a qual 
se destaca a mão de obra qualificada das universidades, representada por mestres e doutores, $e$ também no financiamento da pesquisa e inovação por meio de instituições de fomento. Entre as principais agências de financiamento, é possível destacar a Financiadora de Estudos e Projetos (FINEP), o Banco Nacional de Desenvolvimento Econômico e Social (BNDES), o Conselho Nacional de Desenvolvimento Científico e Tecnológico (CNPq) e a Coordenação de Aperfeiçoamento de Pessoal de Nível Superior (CAPES) (SISTEMA INTEGRADO DE BIBLIOTECAS UNIVERSIDADE DE SÃO PAULO, [2018]). Além disso, a criação de programas e de legislações que reforcem os investimentos e as possibilidades de atuação das ICTs nas atividades de inovação também se caracteriza como ações do SNI. Entre os principais programas de investimentos vale destacar os programas: Fundos Setoriais de Ciência e Tecnologia, Programa de Sustentação do Investimento (PSI-Inovação) e o Inova Empresa (FINEP, 2018b).

Já em relação às legislações que privilegiam o desenvolvimento cientifico e tecnológico e que regulam as atividades de inovação destacam-se o novo Marco Legal - Lei n. 13.243/2016, a qual dispõe sobre estímulos ao desenvolvimento científico, à pesquisa, à capacitação científica e tecnológica e à inovação; a Lei do Bem - Lei n. 11. 196/2005, a qual dispõe sobre os incentivos fiscais para inovação tecnológica; a Lei do Fundo Nacional de Desenvolvimento Científico e Tecnológico (FNDCT), fundamentada pelo Decreto-Lei n. 719/1969, que cria o Fundo Nacional de Desenvolvimento Científico e Tecnológico, e o Decreto n. 9.283/2018, que estabelece medidas de incentivo à inovação e à pesquisa científica e tecnológica no ambiente produtivo, com vistas à capacitação tecnológica, ao alcance da autonomia tecnológica e ao desenvolvimento do sistema produtivo nacional e regional (FINEP, 2018b; BRASIL, 1969; BRASIL, 2005; BRASIL 2016; BRASIL, 2018).

Diante desses esforços direcionados à inovação, é possível delinear as principais estratégias que contribuam com a minimização dos entraves no desenvolvimento e no consequente avanço tecnológico do país. Sendo a avaliação da ascensão do nível de prontidão tecnológica das pesquisas uma das principais estratégias de consolidação do desenvolvimento tecnológico dentro das ICTs, se faz necessário entender os modelos tecnológicos de avaliação e analisar o cenário no qual se inserem as pesquisas brasileiras.

Segundo Pires e Costa Filho (2008), alguns modelos podem servir para avaliar os aspectos críticos referentes ao desenvolvimento de uma tecnologia. Entre os mais comuns, pode-se mencionar o Modelo para Aceitação de Tecnologia (TAM), desenvolvido por Davis em 1989; o Índice de Prontidão para o Uso de Tecnologia (TRI), desenvolvido por Parasuraman em 2000; e a escala Technology Readiness Levels (TRL), desenvolvida pela NASA nas décadas de 1970 e 1980. O modelo TAM é uma adaptação do modelo da Teoria da Ação Raciocinada (TRA), que por ser tão universal, o TRA foi modificado especificamente, para criar modelos de aceitação em tecnologia da informação (SILVA; PIMENTEL; SOARES, 2012). Já o Índice de Prontidão para o Uso de Tecnologia (TRI), que foi desenvolvido para produtos tecnológicos em geral, tem sido testado com resultados generalizáveis para contextos como serviços de seguros e de equipamentos industriais (PIRES; COSTA FILHO, 2008); e, por fim, a escala TRL a qual se destina à avaliação de produtos e processos e os riscos existentes no progresso de desenvolvimento (NASA, 2018).

Em geral, os métodos de avaliação consistem em quantificar os níveis de consolidação das pesquisas e dos produtos finais. Os resultados podem ser interpretados como frutos de um caminho trilhado pela pesquisa básica, posteriormente pela pesquisa aplicada, seguido de 
demonstrações e pilotos e recaindo sobre a entrada no mercado consumidor e da expansão mercadológica (MASSAMBANI, 2017). É importante salientar que não existe um método mais eficaz do que o outro, entretanto, a escala TRL tem sido amplamente utilizada no meio acadêmico em decorrência da facilidade de enquadramento das pesquisas (QUINTELLA, 2017).

Dessa forma, tendo em vista o relevante papel das ICTs como agentes de inovação no Brasil, se faz necessário fortalecer a cultura da interação universidade-empresa-governo no país com intuito de aumentar o nível de maturidade das tecnologias desenvolvidas no âmbito das ICTs. O objetivo deste estudo é apresentar uma análise do panorama das pesquisas realizadas pelas ICTs levando em consideração os principais desafios enfrentados, de modo a indicar os pontos críticos de maior impacto que precisam ser melhorados.

\section{Metodologia}

Este estudo sobre o desenvolvimento tecnológico e a maturidade das pesquisas no âmbito das universidades brasileiras é de caráter exploratório e a metodologia utilizada para a condução do presente estudo foi a indutiva, conduzida por meio da pesquisa bibliográfica, a qual se consolidou por meio da utilização de múltiplas fontes de evidências como artigos em revistas indexadas nas bases Web Of Science, Google Acadêmico, Google Patents e repositórios de instituições de ensino. Além disso, foram exploradas legislações indexadas em sítios governamentais e projetos de programas de incubação da Universidade de Brasília.

O referido estudo desenvolveu-se ainda baseado na análise das tendências das atividades de pesquisa e desenvolvimento em relação à maturidade tecnológica na esfera de bancada e em consonância com as possíveis parcerias para incremento dos níveis de prontidão tecnológica. Para isso, foram utilizadas as palavras-chave "ICTs", "Tríplice Hélice" e "Maturidade Tecnológica" como forma de refinar os resultados.

Durante o desenvolvimento dos estudos, a metodologia utilizada baseou-se em três perspectivas de prospecção: Análise do cenário nacional e dos investimentos governamentais em projetos de pesquisas e inovação; análise do cenário de pesquisa nas universidades brasileiras e análise de startups geradas no ambiente acadêmico. A análise do cenário nacional e dos investimentos governamentais em projetos de pesquisas e inovação foi concebida analisando as linhas de apoio financeiro existentes dos principais órgãos e agências de fomento destinado às Universidades, sejam essas linhas de apoio financeiro à pesquisa ou destinada ao desenvolvimento das startups nascidas no meio acadêmico. Além disso, a análise da pesquisa realizada nas universidades brasileiras e análise das startups geradas no ambiente acadêmico baseou-se em documentos estatísticos do Conselho Nacional de Desenvolvimento Científico e Tecnológico (CNPq) e da Coordenação de Aperfeiçoamento de Pessoal de Nível Superior (CAPES) no ano de 2017 e na análise da criação do Novo Marco Legal e suas principais interferências para as universidades brasileiras. 


\section{Resultados e Discussões}

As pesquisas desenvolvidas pelas ICTs podem ser caracterizadas como pesquisa pura, ou seja, limitada ou cientificista; ou como pesquisa aplicada, na qual há um desenvolvimento e aplicação além do descobrimento de um fenômeno ou entendimento de uma ação (NICOLAU, 2013). Segundo Prodanov e Freitas (2013), atualmente, as universidades tendem a realizar os dois tipos de pesquisas, porém, percebeu-se uma intensificação em relação ao desenvolvimento das pesquisas aplicadas e, portanto, um direcionamento maior para entender determinado problema e para desenvolver soluções passíveis de reprodução. Esse fato tem colaborado para o crescimento do ciclo de inovação, tendo em vista que a inovação só é de fato reconhecida quando ela gera uma melhoria à sociedade (D'ANJOUR; SILVA, 2016). Entretanto, essa inovação, muitas vezes, sofre um processo de estagnação em decorrência das limitações sofridas pelas ICTs, como falta de recursos para aprimoramento das pesquisas, burocracia nas regulamentações exigidas durante o processo de desdobramento das pesquisas científicas ou mesmo carência de parceria de desenvolvimento, por exemplo, a colaboração de empresas públicas e privadas (FINEP, 2018b; TURCHI; MORAIS, 2017).

Segundo Marcos Cintra, presidente da Financiadora de Estudos e Projetos (FINEP), em entrevista à Agência Senado, apesar de todos os problemas, no ano de 2016, o Brasil ocupava a $15^{\circ}$ posição no ranking de produção de conhecimento científico no mundo (SENADO FEDERAL, 2016). Contudo, mesmo ocupando esse expressivo resultado, o nível de prontidão tecnológica apresentado pelas pesquisas desenvolvidas no âmbito das ICTs estaria abaixo do que seria ideal para comercialização (QUINTELLA, 2017). Reforçando a fala de Marcos Cintra, os dados do ranking de produção de conhecimento científico estão contidos no estudo Research in Brazil, apresentado à Coordenação de Aperfeiçoamento de Pessoal de Nível Superior (CAPES), esse estudo analisou diversas publicações entre os anos de 2011 e 2016 (FERREIRA, 2018).

De acordo com Ferreira (2018), não há dúvidas de que a capacidade de geração de pesquisa científica pelas Instituições Científicas, Tecnológicas e de Inovação (ICT) brasileiras, entre elas muitas universidades, é amplamente conhecida no Brasil e no exterior. Além disso, segundo Ferreira (2018), o relatório aponta que o Brasil possui baixas taxas de colaboração industrial e internacional se comparado a outros países em desenvolvimento:

Países economicamente semelhantes e países vizinhos, como a Argentina, trabalham com abordagens distintas para apoiar pesquisas que poderiam ser mais exploradas pelos formuladores de políticas no Brasil. Uma alternativa mencionada pelo relatório é o aumento da colaboração estratégica para determinados setores entre indústria $e$ academia o que poderia beneficiar o desenvolvimento de manufaturas de alta tecnologia. (CLARIVATE ANALYTICS, 2017 apud FERREIRA, 2018, p. 18)

Diante desse cenário e com o intuito de se alcançar o objetivo final de um processo de inovação, se faz necessário avaliar o índice de desenvolvimento de tais pesquisas para que elas possam transpor barreiras e atingir níveis de maturidade em consonância com a cadeia produtiva. Um dos métodos mais comuns de avaliação tecnológica no meio acadêmico é o modelo Technology Readiness Levels (TRL), pois esse modelo possibilita avaliar todo o caminho percorrido pelas pesquisas, desde a sua concepção nas pesquisas de bases até a sua aplicação no público-alvo (GESTIC, 2017; QUINTELLA, 2017). 
Os níveis de prontidão tecnológica - Technology Readiness Levels (TRL) - são um tipo de sistema de medição usado para avaliar o nível de maturidade de um determinado invento. Cada projeto tecnológico é avaliado em relação aos parâmetros para cada nível de prontidão e recebe uma classificação de TRL com base no andamento do projeto. Existem nove níveis de prontidão tecnológica, em que o nível TRL 1 é o mais baixo (desenvolvimento inicial) e o TRL 9 é o mais alto (desenvolvimento concluído) (NASA, 2018). Além disso, é possível inferir que quanto maior for o nível em que uma tecnologia se classifica mais viável será para as universidades realizarem a transferência da tecnologia.

Quando uma tecnologia apresenta TRL entre 1 e 2 , significa dizer que a pesquisa científica está em sua fase inicial e que esses resultados estão sendo traduzidos em pesquisa e desenvolvimento futuros. Geralmente, esse nível de maturidade se relaciona com a concepção da tecnologia, em que se reflete um problema e aspira-se uma possível solução. Essa fase de maturidade é muito especulativa, pois há pouca ou nenhuma prova experimental de conceito para a tecnologia. Além disso, nesse estágio de desenvolvimento, a presença das empresas atuando como apoiadoras do processo de pesquisa são quase inexistentes, cabendo as ICTs um trabalho solitário (NASA, 2018; QUINTELLA, 2017). Já as tecnologias que possuem TRL 3 trazem consigo estudos analíticos e laboratoriais necessários para verificar se uma tecnologia é viável e pronta para prosseguir por meio do processo de desenvolvimento. Muitas vezes, durante o TRL 3, é construído um modelo de prova de conceito. Nesse momento também é possível se observar no meio acadêmico a pesquisa científica básica e parcialmente aplicada (resumos em eventos, artigos, etc.); tradicionalmente denominada de fase de bancada (NASA, 2018; QUINTELLA, 2017). À medida que as pesquisas caminham direcionadas a uma maior maturidade tecnológica, a possibilidade de se prospectar empresas para auxiliar no processo de desenvolvimento aumenta (D'ANJOUR; SILVA, 2016).

As tecnologias que apresentam TRL igual ou acima de TRL 4 começam a se diferenciar de uma simples pesquisa, tornando-se assim uma possibilidade mais real de se tornarem um produto passível de comercialização. Uma vez que a prova de conceito esteja pronta e a tecnologia avança para a TRL 4 pode-se aspirar por uma parceria entre a universidade e indústria com intuito de aprimorar os resultados já alcançados ou mesmo propiciar o seu processo de escalonamento. A TRL 5 é uma continuação da TRL 4, no entanto, uma tecnologia que está em 5 é identificada como uma tecnologia breadboard, ou seja, existe o desenvolvimento tecnológico focado em pesquisa aplicada. Além disso, a tecnologia deve apresentar resultados mais viáveis e mais próximos ao seu consumidor final do que as tecnologias que estão apenas na TRL 4 (NASA, 2018; QUINTELLA, 2017). De acordo com Quintella (2017) nesse estágio de desenvolvimento a transferência de tecnologia tem ainda grande viés acadêmico requerendo rodadas de negociação de Portfólios de PI e mentorias, startups; tradicionalmente denominada de piloto, por exemplo.

Os próximos níveis de maturidade tecnológica (TRL 5 até 9) se destinam as tecnologias com um bom desenvolvimento do produto. Nesses níveis de maturidade, existe maior probabilidade de transferencia de tecnologias por parte das universidades, pois essas correspondem a finalização das tecnologias, com alta interação com incubadoras, parques tecnológicos e aceleradoras (QUINTELLA, 2017). 
De acordo Mazzucato (2014 apud FERREIRA, 2018), sob a perspectiva de aumentar os níveis de prontidão apresentados, destaca-se que o Estado desempenha um importante papel como agente empreendedor na geração e incentivo ao desenvolvimento de inovações tecnológicas, assumindo assim além da responsabilidade de corrigir falhas de mercado o papel de parceiro fundamental do setor privado, facilitando o crescimento e assumindo riscos maiores, normalmente evitados pelas grandes empresas.

Sobre a compreensão "empresas-governo-academia" pode-se referenciar o modelo da Tripla Hélice, desenvolvido por Etzkowitz e Leydesdorff, em que o tripé de desenvolvimento estabelecido por esses três atores promovem um sistema de inovação completo e com maior possibilidade de sucesso (FERREIRA, 2018). Segundo Etzkowitz (2009 apud FERREIRA, 2018):

A universidade é o princípio gerador das sociedades fundadas no conhecimento, assim como o governo e a indústria são as instituições primárias na sociedade industrial. A indústria permanece como o ator-chave e lócus de produção, sendo o governo a fonte de relações contratuais que garantem interações estáveis e o intercâmbio. A vantagem competitiva da universidade em relação a outras instituições de produção do conhecimento são seus estudantes. O fluxo contínuo de admissão e graduação de alunos traz o aporte de novas ideias, em contraste com as unidades de pesquisa e desenvolvimento de laboratórios de empresas e do governo, que tendem a se ossificar, perdendo o "fluxo de capital humano" que é constituído na universidade. (ETZKOWITZ, 2009 apud FERREIRA, 2018, p. 35)

O domínio da pesquisa científica pelas universidades e institutos de pesquisa une forças à capacidade produtiva da indústria e ao fomento estratégico do Estado. A intenção é que dessa união surjam ambientes de inovação relacionando ciência, tecnologia, pesquisa e desenvolvimento tecnológico, de forma contínua e sólida. (ETZKOWITZ, 2009 apud FERREIRA, 2018, p. 35)

Ademais, a base curricular mínima de ensino necessita de mudanças conceituais que proporcionem que o aluno tenha o acesso a noções básicas de empreendedorismo; $e$, consequentemente, possibilitem à academia ampliar seus horizontes de atuação e não somente se fechando num eterno gerador de artigos (QUINTELLA, 2017).

É importante destacar que quando se consegue alinhar a necessidade do mercado com os investimentos com menos morosidade é possível conseguir sucesso no desenvolvimento tecnológico. Um exemplo desse tipo de parceria bem consolidada ocorreu nos estudos sobre o Zika vírus, em que pesquisadores brasileiros integrantes de ICTs foram responsáveis pela investigação a respeito da ligação entre a infecção do vírus e a manifestação da doença em microcefalia (SENADO FEDERAL, 2016). Essa resposta rápida das pesquisas corrobora que os investimentos quando bem empregados, num curto espaço de tempo podem facilitar inclusive a relação das ICTs com o setor empresarial para possíveis parcerias de desenvolvimento, tornando assim o modelo tripla hélice integralizado (SENADOR FEDERAL, 2016; LIMA et al., 2017).

Ainda sob essa reflexão, Quintella (2017) relata também que a produção de artigos e patentes pelas ICTs caracteriza-se como produções intelectuais diferentes e podem se completar sem que um tenha uma valoração maior do outro. Ambas as produções intelectuais possuem avaliações diferentes, grau de maturidades diferentes e cujo reflexo gera a inovação. De acordo com Quintell (2017), artigo e patentes podem ser complementares, e observando bem as limitações para não se infligir os requisitos patentários, tais produções são necessárias para 
se alcançar o parque industrial. E, em uma análise mais profunda, é possível se observar que países de primeiro mundo financiam esse caminho entre artigo e patente por meio do governo e não por meio de empresas. As empresas só iniciam seus investimentos quando a tecnologia de fato está consolidada em decorrência dos possíveis grandes perdas que essas podem sofrer (QUINTELLA, 2017).

Segundo o Senado Federal (2016), em entrevista com Mario Borges, presidente do Conselho Nacional de Desenvolvimento Científico e Tecnológico (CNPq), destaca-se a boa qualidade da pesquisa científica no país. Mas ressalta-se quanto os recursos do Fundo Nacional de Desenvolvimento Científico e Tecnológico (FNDCT) são importantes para desenvolver os programas de ciência e tecnologia \& inovação (CT\&I), configurando, assim, o papel primordial do Estado no processo produtivo.

Segundo Ferreira (2018), os NITs são os principais agentes da universidade empreendedora, pois atuam de forma estratégica dentro do Modelo da Hélice Tríplice ao ligarem as universidades aos governos e às empresas:

Um NIT atuante e bem estruturado é capaz de inserir a ICT em um contexto muito mais amplo e completo, com maiores possibilidades de formalização de parcerias estratégicas, desenvolvimento de projetos inovadores e transferência de tecnologia e conhecimento. Todavia, a ação unilateral visando a disseminação do pensamento empreendedor não é suficiente para que o ambiente de fato se torne mais inovador e evolua para o que é apresentado no Modelo da Hélice Tríplice [...] de nada adianta os índices de proteção de ativos intangíveis das universidades ser elevado se não existe uma cooperação estabelecida com o setor produtivo para a absorção adequada daquelas tecnologias. (FERREIRA, 2018, p. 38)

Um exemplo de estratégia de parceria entre o setor produtivo e as ICTs é a Rede de Centros de Inovação em Tecnologia da Informação e Comunicação (TIC). Segundo a Rede Nacional de Ensino e Pesquisa (2018), essa associação faz parte do Sistema Brasileiro de Tecnologia (SIBRATEC), do Ministério da Ciência, Tecnologia e Inovação (MCTI), operado pela Financiadora de Estudos e Projetos (FINEP), cujo principal objetivo é impulsionar o desenvolvimento tecnológico e econômico do setor de TIC no Brasil.

De acordo com a Rede Nacional de Ensino e Pesquisa (2018), embora o setor empresarial seja o efetivo demandante por inovações, são as ICTs que as desenvolvem. Além disso, o papel das ICTs destina-se a discutir com o setor empresarial as demandas tecnológicas oriundas do mercado e, consequentemente, as possíveis soluções ou aprimoramento para as tecnologias já existentes.

Segundo a Rede Nacional de Ensino e Pesquisa (2018), a Rede de Centros de Inovação em Tecnologias Digitais de Informação e Comunicação (REDETIC) é composta de 30 ICTs, eentre as quais cinco compõem o núcleo de coordenação, sendo uma delas, a RNP, responsável pela gestão administrativa da rede conforme Figura 1. 
Figura 1 - Composição REDETIC

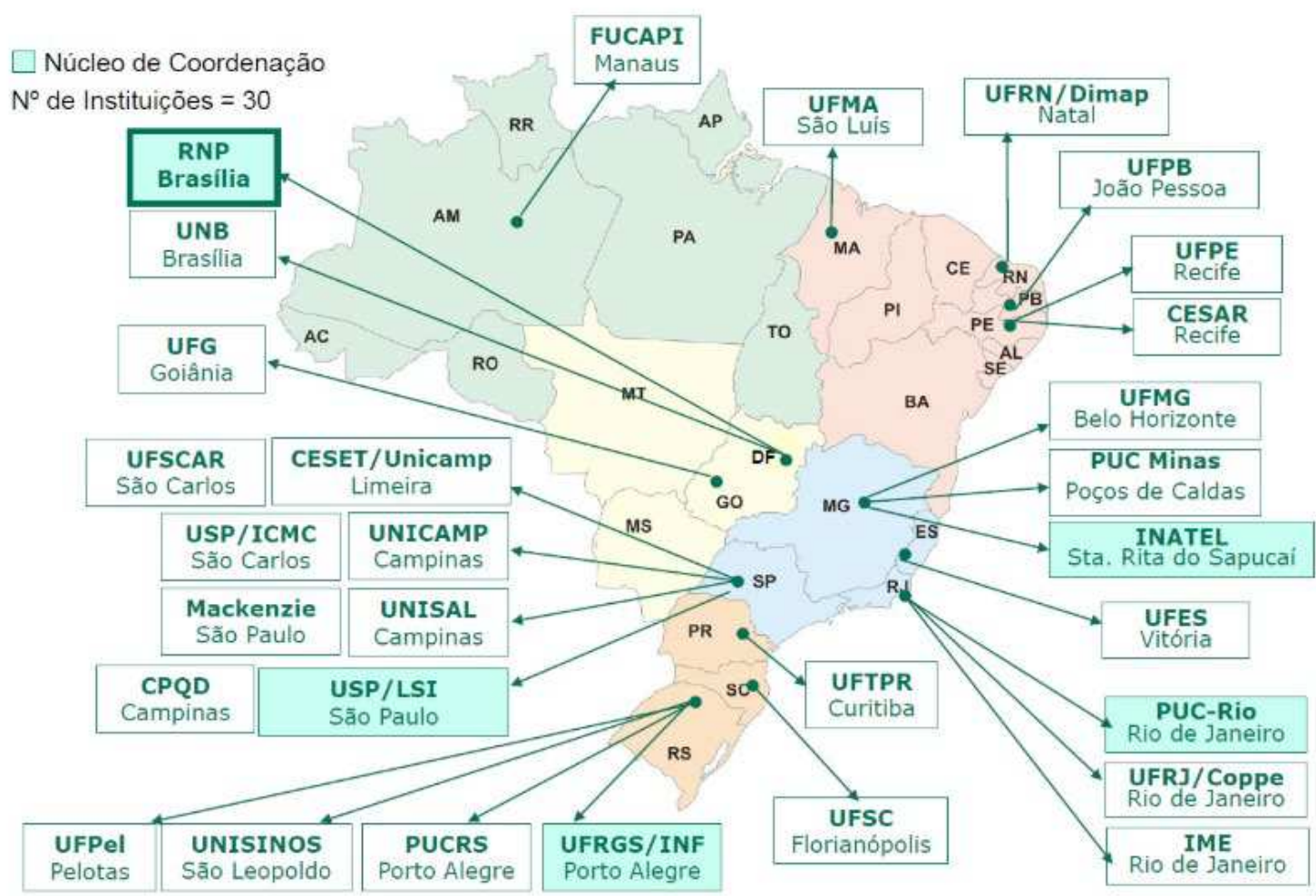

Fonte: Rede Nacional de Ensino e Pesquisa (2018b)

Outro exemplo de parceria para o processo de inovação entre ICTs e empresas são as Startups. De acordo com o Spínola e Brito (2018c), startup em seu conceito aberto são empreendimentos que contemplam a concepção de um produto inovador, com característica repetível e, consequentemente, sujeito ao escalonamento. Geralmente, as startups surgem em cenários incertos, porém, se bem trabalhados, podem se tornar grandes negócios com um mercado consumidor ascendente.

Nesse contexto de inovação, observa-se o surgimento de várias startups no ambiente acadêmico, em especial direcionadas ao setor de base tecnológica. Esse próspero crescimento pode ser atribuído ao estímulo cada vez mais precoce ao empreendedorismo durante os cursos de graduação e de pós-graduação (STEFANO, 2017). Sendo a universidade um celeiro de ideias, a possibilidade de surgimento desse tipo de segmento se torna cada vez mais comum. Além disso, a criação de "startups universitárias" tem se revelado uma saída de crescimento e de amadurecimento de tecnologias antes trabalhadas apenas em bancadas, mas que longe da estagnação trazem consigo infinitas possiblidades de exploração e de consolidação no mercado, agindo, assim, como um vetor de transformação dos modelos tradicionais (STEFANO, 2017).

Nesse contexto, é relevante destacar que o surgimento das startups dentro das universidades abre caminho para a prospecção de novos editais destinados a esse seguimento, como é o caso do programa Finep Startups e também dos programas destinados a micro e pequenas empresas. Normalmente, os editais de fomento à inovação destinados às pesquisas de bases não contemplam o mesmo recurso dos editais destinados às empresas de base tecnológica, sendo atribuídos valores menores quando destinados às pesquisas nas universidades (STEFANO, 2017). O programa Finep Startups busca objetivo apoiar a inovação em empresas nascentes 
intensivas em conhecimento por meio do aporte de recursos financeiros para execução de seus planos de crescimento. Segundo Finep (2018a), o foco do Programa é cobrir o gap de apoio e financiamento existente entre o aporte feito por programas de aceleração, investidores-anjo e ferramentas de financiamento coletivo (crowdfunding) e o aporte feito por Fundos de Seed Money e Venture Capital. O público-alvo atendido pelo programa visa a privilegiar startups que atendam os seguintes quesitos:

- Empresa inovadora cujo produto, processo ou serviço, objeto principal da captação de recursos, esteja no mínimo na fase de protótipo ou testes, preferencialmente já tendo sido realizadas as primeiras vendas;

- Apresentem, no ano do lançamento do Edital, receita bruta de até $\mathrm{R} \$ 3,6$ milhões;

- Tenham sido registradas na Junta Comercial no mínimo seis meses antes do lançamento do Edital; $e$

- Sejam atuantes nos temas e tecnologias habilitadoras previstos no Edital. (FINEP, 2018a)

Além do programa Finep Startups, outro programa com relevante importância para as startups é o Programa de Aceleração InovAtiva Brasil. Segundo a InovAtiva Brasil (2017), o programa consiste em um programa de aceleração em larga escala para negócios inovadores em diversas áreas. O referido programa é idealizado e realizado pelo Ministério da Indústria, Comércio Exterior e Serviços (MDIC) e pelo Serviço Brasileiro de Apoio às Micro e Pequenas Empresas (SEBRAE), com execução da Fundação Centros de Referência em Tecnologias Inovadoras (CERTI). Além disso, conta como parceiro estratégico a Confederação Nacional da Industria (SENAI). De acordo com a InovAtiva Brasil (2017), entre os anos de 2013 e 2017 mais de 640 startups foram aceleradas pelo programa.

No Brasil existem vários órgãos e agências de fomento. Entre as principais, é possível estacar a Financiadora de Estudos e Projetos (FINEP), o Banco Nacional de Desenvolvimento Econômico e Social (BNDES), o Conselho Nacional de Desenvolvimento Científico e Tecnológico (CNPq), a Coordenação de Aperfeiçoamento de Pessoal de Nível Superior (CAPES) e o Serviço Brasileiro de Apoio às Micro e Pequenas Empresas (SEBRAE). Esse conjunto de instituições privilegiam desde o fomento às pesquisas nas universidades, como é o caso da Finep, CNPq e Capes, até o auxílio ao setor empresarial, como é o caso do Sebrae e BNDES.

Em relação aos investimentos proporcionados pela Finep, destacam-se quatro iniciativas de fomento: o Programa Finep Conecta; o Sistema Brasileiro de Tecnologia (SIBRATEC); os Fundos Setoriais; e o Programa de Sustentação do Investimento (PSI). Além dessas alternativas de investimentos, ICTs e Startups podem contar com outros programas específicos, como os editais personalizados, que, em geral, são lançados em momentos oportunos de empreendimentos e pesquisas como é o caso do programa Finep Startups (FINEP, 2018a; FINEP, 2018b; FINEP, 2018c; FINEP, 2018d; FINEP, 2018e).

O Programa Finep Conecta é um programa de apoio à cooperação entre ICT-Empresa, no qual as instituições de pesquisa $e$ as universidades possuem grande valor potencial para as empresas, especialmente quanto a recursos humanos de qualidade, infraestrutura de pesquisa avançada e tecnologias disponíveis para transferência e licenciamento (FINEP, 2018b). De acordo com Finep (2018b), esse tipo de programa destina-se a apoiar empresas brasileiras que tenham projetos em parceria com ICTs e que possuam elevado grau de inovação e potencial 
de geração de externalidades. O principal objetivos desse programa é levar o conhecimento gerado nas ICTs para as empresas, promovendo maior alinhamento dos propósitos da ciência nacional às demandas empresariais e, consequentemente, elevar os dispêndios em P\&D com o intuito de incentivar projetos de maior risco tecnológico.

É importante destacar que programas como o Finep Conecta buscam condições de apoio para a pesquisa, desenvolvimento e aperfeiçoamento de produtos e de processos; consultoria e assessoria técnico-científica; ensaios e testes; análise de materiais; análise de propriedades físico-químicas; elaboração e testes de protótipos; serviços ambientais; calibração; exames laboratoriais e metrologia (FINEP, 2018b).

Outro relevante programa de investimento relacionado à promoção entre universidade e empresa é o Programa de Formação de Recursos Humanos em Áreas Estratégicas (RHAE) idealizado pelo Ministério da Ciência, Tecnologia, Inovação e Comunicações (MCTIC) e CNPq. Segundo o CNPq (2018), o RHAE é destinado à inserção de mestres e doutores em empresas privadas, preferencialmente de micro, pequeno e médio porte. Contudo, por razões orçamentárias, os programas financiados com recursos de Fundos Setoriais, estão com as indicações de novas bolsas, temporariamente, suspensas. Isso afeta não só a inserção de novos pesquisadores nos projetos vigentes, como também remanejamentos (substituições, alterações de nível e mudanças de situação por aquisição de vínculo).

De acordo com CNPq (2018), o Programa utiliza um conjunto de modalidades de bolsas de fomento tecnológico, especialmente criado para agregar pessoal altamente qualificado em atividades de Pesquisa e Desenvolvimento (P\&D) nas empresas, além de formar e capacitar recursos humanos que atuem em projetos de pesquisa aplicada ou de desenvolvimento tecnológico:

O RHAE oferecia bolsas de Fixação e Capacitação de Recursos Humanos - Fundos Setoriais (SET) bem como outras bolsas de fomento tecnológico, como a Desenvolvimento Tecnológico e Industrial (DTI), a Especialista Visitante (EV) e a Apoio Técnico em Extensão no País (ATP).

Em sua última e derradeira edição (Chamada 54/2013) foram oferecidas também bolsas Desenvolvimento Tecnológico e Inovação no Exterior - Junior (DEJ) e Sênior (DES), abrindo a possibilidade de apoiar a participação de especialistas no desenvolvimento de projetos de pesquisa, estudos, treinamentos e capacitação em instituições de excelência no exterior, por meio da realização de estágios e cursos. (CNPQ, 2018)

Segundo Mario Borges (apud SENADO FEDERAL, 2016), a "instabilidade" nos repasses para a inovação é mortal para a ciência, porque provoca a descontinuidade nos programas científicos e o desperdício de dinheiro.

Já o Sibratec é um programa instituído por meio do Decreto n. 6.259/07 com a finalidade de apoiar o desenvolvimento tecnológico do setor empresarial nacional (FINEP, 2018c). De acordo com a Finep (2018c), o Sibratec apoia atividades de P\&D voltadas para a inovação em produtos e processos, em consonância com as prioridades das políticas industrial, tecnológica e de comércio exterior. O objetivo final do Sibratec é aumentar a competitividade das empresas brasileiras.

As entidades que compõem o programa são as Redes de Centros de Inovação, as Redes de Serviços Tecnológicos e as Redes de Extensão Tecnológica, porém, entre essas três redes de apoio, destaca-se a Rede de Centros de Inovações, pois o objetivo dessa rede é gerar e trans- 
formar conhecimentos científicos e tecnológicos em inovações de produtos e processos, e os centros de inovação são unidades ou grupos de desenvolvimento pertencentes aos institutos de pesquisa tecnológica ou às universidades, com experiência no desenvolvimento de produtos ou processos em parceria com empresas (FINEP, 2018c).

E, por fim, em relação ao apoio oferecido pela Finep existem os Fundos Setoriais e o Programa de Sustentação do Investimento (PSI), que, por sua vez, caracterizam-se como instrumentos de financiamento de projetos de pesquisa, desenvolvimento e inovação no País. Segundo a Finep (2018d), esse tipo de fomento foi criado com intuito de se tornar fonte complementar de recursos para financiar o desenvolvimento de setores estratégicos para o País:

A criação dos Fundos Setoriais representa o estabelecimento de um novo padrão de financiamento para o setor, sendo um mecanismo inovador de estímulo ao fortalecimento do sistema de C\&T nacional. Seu objetivo é garantir a estabilidade de recursos para a área e criar um novo modelo de gestão, com a participação de vários segmentos sociais, além de promover maior sinergia entre as universidades, centros de pesquisa e o setor produtivo.

Os Fundos Setoriais constituem ainda valioso instrumento da política de integração nacional, pois pelo menos $30 \%$ dos seus recursos são obrigatoriamente dirigidos às Regiões Norte, Nordeste e Centro-Oeste, promovendo a desconcentração das atividades de C\&T e a consequente disseminação de seus benefícios. (FINEP, 2018d)

No Brasil, atualmente existem diversas estratégias e políticas de apoio voltadas à inovação tecnológica. Entretanto, somente estratégias não são suficientes para se criar um ecossistema de inovação. É preciso, sobretudo, que essas estratégias venham pautadas em legislações específicas que tratem dos assuntos de desenvolvimento e inovação de forma substancial, de preferência baseada no sistema de Tríplice Hélice. Dessa forma, o ciclo de criação, desenvolvimento e comercialização terá grandes possibilidades de subsistir de forma crescente e harmônica. Entre os principais instrumentos jurídicos destacam-se estes: o novo Marco Legal - Lei n. 13.243/2016; a Lei do Bem - Lei n. 11.196/2005; e o Decreto n. 9.283/2018.

O novo Marco Legal - Lei n. 13.243/2016 se caracteriza como um recente instrumento para inovação. A referida lei dispõe sobre estímulos ao desenvolvimento científico, à pesquisa, à capacitação científica e tecnológica e à inovação. Segundo Escobar (2016), por meio dessa lei, universidades públicas e empresas privadas poderão trabalhar de forma muito mais próxima. Ademais, ela permite, entre outras novidades, que professores em regime de dedicação integral desenvolvam pesquisas dentro de empresas e que laboratórios universitários sejam usados pela indústria para o desenvolvimento de novas tecnologias em ambos os casos, com remuneração. Outro aspecto relevante levantado pela nova lei se destina à desburocratização dos sistemas de licitação, compra e importação de produtos relacionados à pesquisa científica e tecnológica (ESCOBAR, 2016).

Assim como o novo Marco Legal, outro importante instrumento jurídico que pretende promover o crescimento do país é a Lei do Bem - Lei n. 11.196/2005. A referida lei dispõe sobre os incentivos fiscais para inovação tecnológica, criando assim concessão de incentivos fiscais às pessoas jurídicas que realizarem pesquisa e desenvolvimento de inovação tecnológica (LEI DO BEM, 2018). Segundo a Lei do Bem (2018), de forma similar ao novo Marco Legal, o governo federal, por meio do MCTIC, utiliza o mecanismo da Lei do Bem para incentivar 
investimentos em inovação por parte do setor privado. Com isso, busca aproximar as empresas das universidades e dos institutos de pesquisa, potencializando os resultados em P\&D. A referida legislação possibilita a atuação P\&D subdividida em três grupos: Pesquisa Básica ou fundamental, Pesquisa Aplicada e Desenvolvimento experimental.

E, por fim, o Decreto n. 9.283/2018 estabelece medidas de incentivo à inovação e à pesquisa científica e tecnológica no ambiente produtivo, com vistas à capacitação tecnológica, ao alcance da autonomia tecnológica e ao desenvolvimento do sistema produtivo nacional $e$ regional. Segundo Matos (2018), o Decreto n. 9.283 possibilitou a alteração de uma série de leis que compõe o Marco legal da Ciência e Tecnologia. O decreto em questão prevê impactos significativos para o empreendedorismo, a inovação e a startup, assim como trouxe também privilégios às universidades e aos centros de pesquisa públicos, às agências de fomento, às empresas públicas e às sociedades de economia mista para participarem como sócias minoritárias do capital social de empresas inovadoras, seja diretamente ou por meio de fundos de investimentos. Tal abertura proporcionada pelo decreto possibilitará às ICTs, em especial as universidades, a oportunidade de vislumbrar um ambiente promissor em relação ao crescimento do nível de prontidão tecnológico de suas tecnologias.

\section{Considerações Finais}

Diante do exposto, depreende-se que o panorama de inovação no Brasil ainda está sendo construído. Apesar de ser a maior economia da América Latina, o Brasil ainda é superado por países como Chile, Colômbia e Uruguai. Destinar investimentos desde o primeiro momento das pesquisas se faz crucial na corrida do desenvolvimento tecnológico. Enquanto os países desenvolvidos investem cada vez mais em inovação, pois entendem que essa é a estratégia mais segura para ser e permanecer desenvolvidos. Convive-se com recursos contingenciados, intensa burocracia e um ambiente de negócios hostil à inovação no Brasil. Contudo, esforços direcionados à junção entre ICTs, setor empresarial e governo podem contribuir para um futuro promissor e um contínuo crescimento do país.

\section{Referências}

BRASIL. Código Civil. Decreto-Lei n. 719. Cria o Fundo Nacional de Desenvolvimento Científico e Tecnológico e dá outras providências. Diário Oficial [da] República Federativa do Brasil, Brasília, DF, 31 julho de 1969. Disponível em: <http:/www.planalto.gov.br/ccivil_03/decreto-lei/ Del0719.htm>. Acesso em: 27 jun. 2018.

Código Civil. Lei n. 10.973, de 2 de dezembro de 2004. Dispõe sobre incentivos à inovação e à pesquisa científica e tecnológica no ambiente produtivo e dá outras providências. Diário Oficial [da] República Federativa do Brasil, Brasília, DF, 3 dezembro de 2004. Disponível em: < http:// www.planalto.gov.br/ccivil_03/_ato2004-2006/2004/lei/110.973.htm>. Acesso em: 26 jun. 2018.

Código Civil. Lei n. 11. 196, de 21 de novembro de 2005. Instituí o Regime Especial de Tributação para a Plataforma de Exportação de Serviços de Tecnologia da Informação - Repes.

Diário Oficial [da] República Federativa do Brasil, Brasília, DF, 22 de novembro de 2005. Disponível em: <http://www.planalto.gov.br/ccivil_03/_ato2004-2006/2005/lei/111196.htm>. Acesso em: 27 jun. 2018. 
Código Civil. Lei n. 13.243, de 11 de janeiro de 2016. Dispõe sobre estímulos ao desenvolvimento científico, à pesquisa, à capacitação científica e tecnológica e à inovação. Diário Oficial [da] República Federativa do Brasil, Brasília, DF, 12 de janeiro de 2016. Disponível em: < http://www.planalto.gov.br/ccivil_03/_ato2015-2018/2016/lei/113243.htm>. Acesso em: 27 jun. 2018.

Código Civil. Decreto-Lei n. 9.283, de 7 de fevereiro de 2018. Estabelecer medidas de incentivo à inovação e à pesquisa científica e tecnológica no ambiente produtivo, com vistas à capacitação tecnológica, ao alcance da autonomia tecnológica e ao desenvolvimento do sistema produtivo nacional e regional. Diário Oficial [da] República Federativa do Brasil, Brasília, DF, 8 de fevereiro de 2018. Disponível em: <http:/www.planalto.gov.br/ccivil_03/_Ato2015-2018/2018/ Decreto/D9283.htm>. Acesso em: 27 jun. 2018.

CNPq - CONSELHO NACIONAL DE DESENVOLVIMENTO CIENTÍFICO E TECNOLÓGICO. Rhae. Brasília, [2018]. Disponível em: <http://cnpq.br/apresentacao-rhae>. Acesso em: 27 jun. 2018.

D'ANJOUR, Miler Franco; SILVA, Napiê Galvê Araújo. Mensurando a inovação: avaliação em mpes participantes do programa agentes locais de inovação. Rio Grande do Norte: SEBRAE, 2016. Disponível em: <https://m.sebrae.com.br/Sebrae/Portal\%20Sebrae/UFs/RN/Anexos/Livro_artigos_ digital_NET.pdf $>$. Acesso em: 28 jun. 2018.

ESCOBAR, Herton. Marco legal de ciências e tecnologia: o que muda na vida dos pesquisadores? Estadão, 2016. Disponível em: <https://ciencia.estadao.com.br/blogs/herton-escobar/marco-legalde-ciencia-e-tecnologia-o-que-muda-na-vida-dos-pesquisadores/>. Acesso em: 24 jun. 2018.

FERREIRA, Camila Lisdália Dantas. A hélice tríplice e a universidade de Brasília: as atividades de transferência de tecnologia conduzidas pelo núcleo de inovação tecnológica. 2018. 119 f. Dissertação (Mestrado em Propriedade Intelectual e Transferência de Tecnologia para Inovação) Universidade de Brasília: Profnit, 2018.

FINEP - EMPRESA BRASILEIRA DE INOVAÇÃO E PESQUISA. Finep Startup. Rio de Janeiro, [2018a]. Disponível em: <http://www.finep.gov.br/apoio-e-financiamento-externa/programas-elinhas/finep-startup>. Acesso em: 28 jun. 2018.

Programa Finep Conecta: Apoio à Cooperação ICT-Empresa. Rio de Janeiro, [2018b]. Disponivel em: <http://www.finep.gov.br/apoio-e-financiamento-externa/programas-e-linhas/finepconecta >. Acesso em: 28 jun. 2018.

Sibratec. Rio de Janeiro, [2018c]. Disponível em: <http://www.finep.gov.br/apoio-efinanciamento-externa/programas-e-linhas/sibratec >. Acesso em: 28 jun. 2018.

O que são fundos setoriais. Rio de Janeiro, [2018d]. Disponível em: <http://www.finep. gov.br/a-finep-externo/fontes-de-recurso/fundos-setoriais/o-que-sao-fundos-setoriais $>$. Acesso em 28 jun. 2018.

PSI - Programa de sustentação do investimento. Rio de Janeiro, [2018e]. Disponível em: <http://www.finep.gov.br/a-finep-externo/fontes-de-recurso/outras-fontes/psi-programa-desustentacao-do-investimento >. Acesso em: 26 jun. 2018.

GESTIC, Patrícia Leal. Patentes de universidade mais atrativas para empresas. Inova Unicamp: Campinas, 2017. Disponível em: < https://www.inova.unicamp.br/noticia/patentes-de-universidademais-atrativas-para-empresas/>. Acesso em: 27 jun. 2018. 
INOVATIVA BRASIL. Programa de Aceleração Inovativa Brasil. Brasília, 2017. Disponível em: $<$ https://www.inovativabrasil.com.br/>. Acesso em: 28 de jun. 2018.

LEI DO BEM. Lei do bem. São Paulo, [2018]. Disponível em: <http://www.leidobem.com/lei-dobem-inovacao/>. Acesso em: 26 jun. 2018.

LIMA, Larisse Araújo et al. Sinopse do cenário cervejeiro: o advento da produção e o mercado na região centro oeste. Cadernos de prospecção, Rio de Janeiro, v. 10, p. 650-664, 2017. Disponível em: <https://portalseer.ufba.br/index.php/nit/article/view/23041>. Acesso em: 28 jun. 2017.

MASSAMBANI, Oswaldo. Estratégias da FINEP e seus instrumentos de apoio à inovação. São Paulo, 2017. Disponível em: <http://www.desenvolvesp.com.br/wp-content/uploads/2017/05/FINEP_ MPI_2017.pdf $>$. Acesso em: 28 jun. 2018.

MATOS, Felipe. Universidades Públicas e órgão do governo poderão ser sócios de startups. Estadão, São Paulo, 2018. Disponível em: < https://link.estadao.com.br/blogs/felipe-matos/universadespublicas-e-orgaos-do-governo-poderao-ser-socios-de-startups/>. Acesso em: 27 jun. 2018.

NASA - NATIONAL AERONAUTICS AND SPACE ADMINISTRATION. Technology Readiness

Level. Washington, 2018.Disponível em: < https://www.nasa.gov/directorates/heo/scan/engineering/ technology/txt_accordion1.html>. Acesso em: 27 jun. 2018.

NICOLAU, Marcos. Pesquisa aplicada à comunicação em mídias digitais - DEMID. Paraíba, 2013. Disponível em: < http://www.insite.pro.br/elivre/pesquisa_cientifica_midiasdigitais.pdf > . Acesso em: 28 jun. 2018.

PIRES, Péricles José; COSTA FILHO, Bento Alves da. Fatores do índice de prontidão à tecnologia (TRI) como elementos diferenciadores entre usuários e não usuários de internet banking e como antecedentes do modelo de aceitação de tecnologia (TAM). RAC, Curitiba, v. 12, n. 2, p. 429-456, abr/jun., 2008. Disponível em: <http://www.scielo.br/pdf/rac/v12n2/a07v12n2.pdf > . Acesso em: 28 jun. 2018.

PRODANOV, Cleber Cristiano; FREITAS, Ernani Cesar de. Metodologia do trabalho científico: métodos e técnicas da pesquisa e do trabalho acadêmico. ed. Novo Hamburgo: Feevale, 2013, 277 f. 2. Disponível em: <http:/www.feevale.br/Comum/midias/8807f05a-14d0-4d5b-b1ad-1538f3aef538/Ebook\%20Metodologia\%20do\%20Trabalho\%20Cientifico.pdf>. Acesso em: 28 jun. 2018.

QUINTELLA, C. M. A revista caderno de prospecção e os níveis de maturidade de tecnologias (TRL). Cadernos de Prospecção, Salvador, v. 10, n. 1-2, p. 1, jan./mar., 2017. Disponível em: $<$ https://portalseer.ufba.br/index.php/nit/article/view/21864/pdf_202>. Acesso em: 27 jun. 2018.

REDE NACIONAL DE ENSINO E PESQUISA - RNP. Instituições de Ciência e Tecnologias (ICTs). Brasília, DF, 2018a. Disponível em: <http://www.redetic.rnp.br/redetic/instituicoes-de-cienciae-tecnologia-icts/>. Acesso em: 26 jun. 2018.

REDE NACIONAL DE ENSINO E PESQUISA - RNP. Sobre a REDETIC. Brasília, DF, 2018b. Disponível em <http://www.redetic.rnp.br/sobre-a-redetic/>. Acesso em: 28 jun. 2018.

SENADO FEDERAL. Burocracia e falta de recursos emperram produção cientifica, dizem especialistas. Brasília, DF, 2016. Disponível em: <https:/www12.senado.leg.br/noticias/ materias/2016/11/22/burocracia-e-falta-de-recursos-emperram-producao-cientifica-dizemespecialistas >. Acesso em: 28 jun. 2018.

SILVA, Patrícia; PIMENTEL, Valdenise; SOARES, Juliana. A utilização do computador na educação: 
aplicando o Technology Acceptance Model (TAM). Biblionline, João Pessoa, v. 8, n. especial, p. 263-272, 2012. Disponível em: <http://www.brapci.inf.br/index.php/article/download/53414>. Acesso em: 28 jun. 2018.

SISTEMA INTEGRADO DE BIBLIOTECAS UNIVERSIDADE DE SÃO PAULO - SIBi. Agências e oportunidades de financiamento. São Paulo, [2018]. Disponível em: <http://www.sibi.usp.br/ apoio-pesquisador/agencias-financiamento/> . Acesso em: 28 jun. 2018.

SPÍNOLA, André; BRITO, Márcio. O que é uma startup. Brasília, c2018. Disponível em: <http:// www.sebrae.com.br/sites/PortalSebrae/sebraeaz/o-que-e-uma-startup,616913074c0a3410VgnVCM1 000003b74010aRCRD>. Acesso em: 4 jul. 2018.

STEFANO, Fabiane. A universidade (com jeito de startup) que quer mudar tudo. Revista Exame: São Paulo, 2017. Disponível em: < https://exame.abril.com.br/revista-exame/a-universidadeque-quer-mudar-tudo/>. Acesso em: 25 jun. 2018.

TURCHI, Lenita; MORAIS, José Mauro de. Políticas de apoio à inovação tecnológica no Brasil: Avanços recentes, limitações e propostas de ações. Brasília: IPEA, 2017. 485f .Disponível em: $<$ http://www.ipea.gov.br/agencia/images/stories/PDFs/livros/livros/171103_politicas_de_apoio_a_ inovacao.pdf $>$. Acesso em: 28 jun. 2018.

\section{Sobre os autores}

\section{Grace Ferreira Ghesti}

E-mail: ghesti.grace@gmail.com

Doutora em Química pela Universidade de Brasília (2009). Mestra pelo Programa de Mestrado Profissionalizante em Certified Brewmaster Course Versuchs- und Lehranstalt für Brauerei in Berlin, VLB, Alemanha (2008). Mestra em Química pela Universidade de Brasília (2006). Bacharel em Química pela Universidade de Brasília (2004). Endereço Profissional: Campus Universitário Darcy Ribeiro, Universidade de Brasília, Instituto de Química (IQ). UnB, Brasília, DF. CEP: 70297-400.

\section{Larisse Araújo Lima}

E-mail: larissealima@gmail.com

Mestre em Ciências de Materiais Nanoestruturados (UnB). Graduada em Química (2009).

Endereço profissional: Universidade de Brasília, Centro de Apoio ao Desenvolvimento Tecnológico (CDT/UnB).

Campus Universitário Darcy Ribeiro, Edifício CDT, Brasília, DF. CEP: 70904-970.

\section{Lincoln Pinheiro de Oliveira}

E-mail: pinheiro.lincoln@hotmail.com

Engenheiro químico (UFVJM) e Mestre em Tecnologias Química e Biológica (UnB)

Endereço profissional: Universidade de Brasília, Centro de Apoio ao Desenvolvimento Tecnológico (CDT/UnB).

Campus Universitário Darcy Ribeiro, Edifício CDT, Brasília, DF. CEP: 70904-970.

\section{Luiza Xavier da Silva Tenório}

E-mail: luiza.xavier.st@gmail.com

Mestre em Zoologia e graduada em Ciências Biológicas pela Universidade de Brasília (UnB). 
Endereço profissional: Universidade de Brasília, Centro de Apoio ao Desenvolvimento Tecnológico (CDT/UnB). Campus Universitário Darcy Ribeiro, Edifício CDT, Brasília, DF. CEP: 70904-970.

\section{Marcio Lima da Silva}

E-mail: dasilva.marciolima@gmail.com

Doutor em Mecânica dos Fluidos, Energética e Processos pela Universidade de Rhones-Alpes, França (2014). Engenheiro Mecânico pela Universidade de Brasília (2010).

Endereço profissional: Universidade de Brasília, Centro de Apoio ao Desenvolvimento Tecnológico (CDT/UnB). Campus Universitário Darcy Ribeiro, Edifício CDT, Brasília, DF. CEP: 70904-970.

\section{Sarah Sampaio Py-Daniel}

E-mail: sarahpydaniel@gmail.com

Mestre em Ecologia (2017). Graduada em Ciências Biológicas com ênfase em Biodiversidade e Conservação (2013).

Endereço profissional: Universidade de Brasília, Centro de Apoio ao Desenvolvimento Tecnológico (CDT/UnB). Campus Universitário Darcy Ribeiro, Edifício CDT, Brasília, DF. CEP: 70904-970.

\section{Thiago Lara Fernandes}

E-mail: thiagolarafernandes@hotmail.com

Graduado em Química pela Universidade Católica de Brasília (2015).

Endereço profissional: Universidade de Brasília, Centro de Apoio ao Desenvolvimento Tecnológico (CDT/UnB). Campus Universitário Darcy Ribeiro, Edifício CDT, Brasília, DF. CEP: 70904-970. 